\title{
The Quest for Light Sea Quarks: Algorithms for the Future
}

\author{
W. Schroers ${ }^{\mathrm{a}}$, N. Eicker ${ }^{\mathrm{a}}$, M. D'Elia ${ }^{\mathrm{b}}$, Ph. de Forcrand ${ }^{\mathrm{c}}$, C. Gebert ${ }^{\mathrm{d}}$, Th. Lippert ${ }^{\mathrm{a}}$, I. Montvay $^{\mathrm{d}}$, \\ B. Orth ${ }^{\mathrm{a}}$, M. Pepe ${ }^{\mathrm{e}}$, K. Schilling ${ }^{\mathrm{a}}$

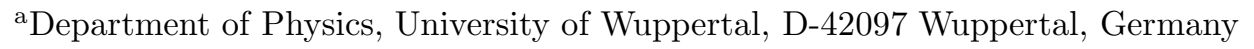

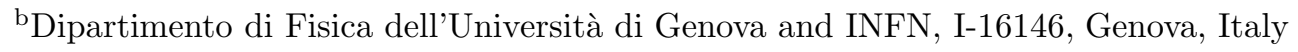 \\ ${ }^{\mathrm{c} I n s t . ~ f u ̈ r ~ T h e o r e t i s c h e ~ P h y s i k, ~ E T H ~ H o ̈ n g g e r b e r g, ~ C H-8093 ~ Z u ̈ r i c h, ~ S w i t z e r l a n d ~}$ \\ dTheory Division, DESY, Notkestr. 85, D-22603 Hamburg, Germany \\ ${ }^{\mathrm{e}}$ Laboratoire de Physique Théorique Université de Paris-Sud, Bâtiment 210 F-91405 Orsay-Cedex
}

\begin{abstract}
As part of a systematic algorithm study, we present first results on a performance comparison between a multibosonic algorithm and the hybrid Monte Carlo algorithm as employed by the SESAM collaboration. The standard Wilson fermion action is used on $32 \times 16^{3}$ lattices at $\beta=5.5$.
\end{abstract}

\section{Introduction}

The past six years have witnessed a number of large simulations of full QCD with two degenerate sea quarks and Wilson like actions. All of them were based on the hybrid Monte Carlo algorithm (HMC) which has been systematically improved over the years. These simulations, however, albeit being milestones for lattice QCD in the pre-Teracomputing age [1], suffer from severe restrictions that prevent them to be truly realistic: we need to go to lighter quark masses and operate with three flavors with masses eventually approaching the physical mass.

This situation has been motivating research and development of alternative algorithms of the multibosonic variety (MBA) that promise in principle to overcome some of the restrictions of HMC mentioned. It has been shown that MBA can indeed be geared to reach the efficiency of HMC [2] and encouraging results have been achieved with MBA for the treatment of finite density QCD [3], supersymmetric field theories [4] as well as the case of three dynamical fermion flavors [5].

In this contribution we wish to describe our benchmarking of MBA, performed in the quest to achieve lighter quark masses than previously

\footnotetext{
*Email: Wolfram.Schroers@Feldtheorie.de
}

possible in the SESAM and other full QCD simulations. For details regarding the HMC algorithm used see ref. [6].

\section{Gauging performance}

The efficiency of an algorithm is most suitably quoted as the computational cost, $E_{\text {ind }}$, for producing a statistically independent vacuum gauge field configuration within the Markov process, expressed in units of the number of necessary Diracmatrix vector $M \cdot \phi$ multiplies:

$E_{\text {ind }}=2 \tau_{\text {int }} E_{\text {traj }}$,

with $\tau_{\text {int }}$ being the integrated autocorrelation time measured in units of update sequences called "trajectories" each having a cost of $E_{\text {traj }}$. Being

\begin{tabular}{cccc}
\hline \multicolumn{4}{c}{ Volume: $\Omega=32 \times 16^{3}, \beta=5.5$} \\
\hline$\kappa$ & $\mathbf{a} / \mathbf{f m}$ & $\mathbf{r}_{\mathbf{0}} / \mathbf{a}$ & $\mathbf{m}_{\pi} / \mathbf{m}_{\rho}$ \\
\hline 0.159 & 0.141 & $4.39(3)$ & $0.800(10)$ \\
0.160 & 0.117 & $4.89(3)$ & $0.670(14)$ \\
\hline
\end{tabular}

Table 1

Working points chosen.

particularly interested in the quark mass dependence of the algorithms, we chose for our analysis 
two different quark mass settings, as given in table 1. Note that both quark masses are from the regime of current full QCD simulations.

\section{Defining the MBA algorithm}

The parameter space of MBA algorithms is large w.r.t. the HMC situation: MBA optimization proceeds (a) by finding a not too high order polynomial approximating the inverse of the fermion matrix at the given working point, (b) by devising an efficient "trajectory" in form of an updating sequence for the degrees of freedom and (c) by exploiting a method to correct for the remaining systematic error. Today, all MBAs use a noisy correction step for (c). For a detailed discussion see e.g. ref. [7,8].

For the construction of appropriate polynomials we followed the prescription proposed in ref. [9.10] which employs the GMRES algorithm to find a non-hermitian approximation of the inverse Wilson matrix with ultraviolet-filtering on a small sample of thermalized gauge field configurations. This was done to minimize the polynomial order and hence the number of boson fields. Quadratically optimized polynomials [1] need about $2-3$ times more boson fields for similar acceptance rates.

The other key element for the optimization of an MBA is the choice of "trajectory", T, i.e., the basic updating pattern. For that matter, one has to devise an appropriate blend of gauge and boson field updates. We have chosen the "trajectory" to be

$T=C O_{g}\left(O_{b} O_{g}\right)^{5} H_{b}\left(O_{g} O_{b}\right)^{5} O_{g}$,

with $C$ being the final accept/reject correction step, $O_{g}\left(O_{b}\right)$ a gauge (boson) field overrelaxation sweep and $H_{b}$ a boson field global quasiheat bath [12].

The number of boson fields is tuned such as to ensure a good acceptance rate in the ball-park of $60-70 \%$. As a result we obtained the values quoted in table 2 .

\section{Results}

The determination of accurate autocorrelation times, $\tau_{\text {int }}$, needs very long Monte Carlo time

\begin{tabular}{lccc}
\hline$\kappa$ & $\mathbf{n}$ & $\mathbf{P}_{\text {acc }}$ & $\boldsymbol{\Delta} \beta$ \\
\hline 0.159 & 24 & $60.3 \%$ & 0.876 \\
0.160 & 42 & $65.9 \%$ & 1.763 \\
\hline Table 2 & & &
\end{tabular}

Table 2

Number of boson fields $n$ required for the MBA with the resulting acceptance rates at the two working points and the shift of the gauge coupling used for UV-filtering.

histories. The number of trajectories underlying the present analysis together with our results for the efficiencies, $E_{\text {ind }}$, are collected in table 1 . At $\kappa=0.160$, we have also used the topological charge, $Q$, as a monitor for autocorrelations.

\begin{tabular}{clcc}
\hline$\kappa$ & Observ. & HMC & MBA \\
\hline 0.159 & Plaq. & $0.55816(4)$ & $0.55804(7)$ \\
& $\left(a m_{\pi}\right)$ & $0.4406(33)$ & $0.4488(37)$ \\
& $\left(a m_{\rho}\right)$ & $0.5507(59)$ & $0.5635(83)$ \\
\hline 0.160 & Plaq. & $0.56077(6)$ & $0.56067(5)$ \\
& $\left(a m_{\pi}\right)$ & $0.3041(36)$ & $0.3184(68)$ \\
& $\left(a m_{\rho}\right)$ & $0.4542(77)$ & $0.4674(81)$ \\
\hline
\end{tabular}

Table 3

Average plaquette, non-singlet pseudo-scalar $\left(a m_{\pi}\right)$ and vector $\left(a m_{\rho}\right)$ meson masses.

As a check for our simulation code we have computed the average plaquette and (non-singlet) pseudo-scalar and vector meson mass values, as shown in table 3. They agree within errors.

$E_{\text {ind }}$ (plaq) has been obtained from direct integration of the plaquette autocorrelation functions while for the masses the jackknife blocking technique has been applied. The latter method only allows for a rather crude estimate of autocorrelation times, so that we concentrate mainly on the plaquette in the following, where we have safe control over statistical errors:

The statistics at the point $\kappa=0.159$ covers about 320 autocorrelation times for the HMC and 140 for the MBA. At the working point $\kappa=0.160$, the HMC series has a length of 140 and the MBA of 160 times $\tau_{\text {int }}$. Hence, the statistics is sufficient for the results to be conclusive. 


\begin{tabular}{clcccccc}
\hline$\kappa$ & Alg. & \# trajectories & $\tau_{\text {int }}($ plaq $)$ & $\mathbf{E}_{\text {ind }}($ plaq $)$ & $\mathbf{E}_{\text {ind }}\left(\mathbf{m}_{\pi}\right)$ & $\mathbf{E}_{\text {ind }}\left(\mathbf{m}_{\rho}\right)$ & $\mathbf{E}_{\text {ind }}(\mathbf{Q})$ \\
\hline 0.159 & HMC & 3521 & $11.0(0.4)$ & $712(26) 10^{3}$ & $<162010^{3}$ & $<162010^{3}$ & \\
& MBA & 6217 & $44.7(3.4)$ & $786(60) 10^{3}$ & $52810^{3}$ & $70410^{3}$ & \\
\hline 0.160 & HMC & 5003 & $34.1(3.1)$ & $572(52) 10^{4}$ & $35010^{4}$ & $89410^{4}$ & $850(34) 10^{4}$ \\
& MBA & 9910 & $61.1(4.1)$ & $216(14) 10^{4}$ & $10210^{4}$ & $7010^{4}$ & $324(22) 10^{4}$ \\
\hline
\end{tabular}

Table 4

Lengths of MC runs, estimated plaquette autocorrelation times and total cost, $E_{\text {ind }}$, for producing one configuration decorrelated w.r.t. various observables, from HMC and MBA.

From table 1 one finds that $E_{\text {ind }}($ plaq) is about equal for $\mathrm{MBA}$ and $\mathrm{HMC}$ at the larger quark mass. The total effort in the case of the HMC increases by a factor of 8 when stepping down in quark mass, while at the same time the cost for MBA 'only' rises by a factor of 2.7. Consequently, the MBA has become almost a factor of three more efficient than HMC.

\section{Conclusions}

We have demonstrated in a realistic setting that MBA-type sampling techniques are not at all inferior to the state-of-the-art HMC techniques and appear to show superior scaling behavior in quark masses. We have established here a better scaling behavior of MBA, leading to a gain factor of almost three in favor of the particular MBA variant at currently reachable quark masses.

It remains to be seen to what extent this encouraging result will pertain in the regime of smaller quark masses. The ultimate aim is to push full QCD simulation with Wilson like fermions below the point $m_{\pi} / m_{\rho} \leq 0.5$.

Another point to check is whether the MBA variant considered in this paper with its nonhermitian approximation will remain operational for practical simulations in the deeper chiral regime. Because the GMRES method suffers from numerical instabilities for increasing orders $n$, one might have to take recourse - even on $32 \times 16^{3}$ lattices - to 128 -bit precision. A more extended MBA study in this direction is in preparation [13].

\section{Acknowledgments}

N.E. is supported under DFG grant Li701/31, M.P. by the European Community's Human potential program under HPRN-CT-2000-00145 Hadrons/Lattice QCD, and W.S. by the DFG Graduiertenkolleg "Feldtheoretische Methoden in der Elementarteilchentheorie und Statistischen Physik". The numerical productions were run on the APE-100 systems installed at NIC Zeuthen.

\section{REFERENCES}

1. Th. Lippert, these proceedings; A. Ukawa, ibid; H. Wittig, ibid.

2. C. Alexandrou et al., Phys. Rev. D 61 (2000) 074503.

3. S. Hands et al., Eur. Phys. J. C 17 (2000) 285.

4. I. Campos et al. [DESY-Münster Coll.], Eur. Phys. J. C 11 (1999) 507.

5. C. Gebert, F. Farchioni, I. Montvay, and W. Schroers, these proceedings.

6. Th. Lippert, Habilitationsschrift, Univ. of Wuppertal, 2001.

7. P. de Forcrand, Parallel Computing 25 (1999) 1341.

8. W. Schroers, PhD-Thesis, Univ. of Wuppertal, 2001.

9. A. Borrelli et al., Nucl. Phys. B 477 (1996) 809.

10. P. de Forcrand, Nucl. Phys. Proc. Suppl. 73 (1999) 822.

11. I. Montvay, Comput. Phys. Commun. 109 (1998) 144.

12. P. de Forcrand, Phys. Rev. E 59 (1999) 3698.

13. W. Schroers, N. Eicker, M. D'Elia, Ph. de Forcrand, C. Gebert, Th. Lippert, I. Montvay, B. Orth, M. Pepe, and K. Schilling, in preparation. 